Potts, H.E. and Diver, D.A. (2007) Automatic recognition and characterisation of supergranular cells from photospheric velocity fields. Solar Physics 248(2):pp. 263-275.

http://eprints.gla.ac.uk/3785/

Deposited on: 15 July 2008 


\title{
Automatic Recognition and Characterisation of Supergranular Cells from Photospheric Velocity Fields
}

\author{
H.E. Potts and D.A. Diver
}

Received 17 May 2007; Accepted 23 September 2007

(C) Springer $\bullet \bullet \bullet \bullet$

\begin{abstract}
We have developed an exceptionally noise resistant method for accurate and automatic identification of supergranular cell boundaries from velocity measurements. Due to its high noise tolerance the algorithm can produce reliable cell patterns with only very small amounts of smoothing of the source data in comparison to conventional methods. In this paper we describe the method and test it with simulated data. We then apply it to the analysis of velocity fields derived from high-resolution continuum data from MDI (Michelson Doppler Imager) on SOHO. From this, we can identify certain basic properties of supergranulation cells, such as their characteristic sizes, the flow speeds within cells and their dependence on cell areas at high resolution. The effect of the noise and smoothing on the derived cell boundaries is investigated and quantified using simulated data. We show in detail the evolution of supergranular cells over their lifetime, including observations of emerging, splitting, and coalescing cells. A key result of our analysis of cell internal velocities is that there is a simple linear relation between cell size and cell internal velocity, rather than the power law usually suggested.
\end{abstract}

Keywords: Photosphere, Supergranulation, Granules, Photospheric Flow

\section{Introduction}

The convection processes in the Sun have been studied for many years, with the first observation of the solar granulation made by William Herschel in 1801 . Granulation is a small-scale, rapid convectional process $(\sim 1 \mathrm{Mm}$ diameter, fewminute lifetime, $1 \mathrm{~km} \mathrm{~s}^{-1}$ typical flow speed) which has been well described and modelled. More recently, larger scale patterns with weaker flows have been observed superimposed on the basic granulation flow. The clearest of these is the supergranular flow, first observed by Hart (1954), and recognised as a cellular flow system by Leighton, Noyes, and Simon (1961) by analysing dopplergrams taken at the Mount Wilson Observatory. Supergranulation is a flow with a cellular form, probably convectional in nature, with characteristic size around $15-30 \mathrm{Mm}$, 20 hour lifetime, and typical flow speeds around $300 \mathrm{~ms}^{-1}$. The supergranular

Department of Physics and Astronomy, University of Glasgow, Glasgow, G12 8QQ, UK. 
pattern is most dramatically seen as patterns in the line-of-sight velocity of the solar surface seen, for example, in full-disk MDI dopplergrams from the SOHO satellite. The velocities in supergranulation, as seen in the photosphere, are predominantly horizontal. A consequence of this is that the supergranulation pattern in dopplergrams is most visible towards the limb of the Sun. In order to observe the supergranulation at higher resolutions and near disk centre, analysis of the horizontal photospheric flow fields is required. Although supergranulation has been observed for nearly 50 years remarkably little is known about it - indeed it is not even certain if it driven by convection, as no significant temperature gradient has been measured across the cells. The best attempts to date at measuring any temperature variation have concluded that it must be less than $3 \mathrm{~K}$ across a cell (Lin and Kuhn, 1992), and only one recent measurement (Meunier, Tkaczuk, and Roudier, 2007) shows a measurable temperature change from cell centre to cell boundary of $0.8-2.8 \mathrm{~K}$, derived from a continuum intensity variation of $0.05-$ $0.18 \%$. Part of the difficulty in analysing such flows is that they are masked by the much more rapidly varying granulation flows which have large temperature variations, and the higher concentration of magnetic fields at cell boundaries.

With the advent of long time series, high-resolution data from satellite missions such as SOHO and TRACE we can now track the motion within these larger structures. When looking at the large scale but weak motions found in supergranulation, it can be hard to interpret the data to identify coherent structures. In this paper we present a new method to construct supergranular boundaries with confidence from flow fields, and once constructed how such structures can be exploited to derive further essential diagnostic information. The article is constructed as follows: Section 2 describes the algorithm for finding and displaying the cell boundaries; Section 3 tests the algorithm against simulated data, and compares results with a conventional divergence algorithm; Section 4 shows how the algorithm works on real solar data; Section 5 contains notes about the potential problems when using the method, and how to avoid them.

\section{Cell Analysis Method}

This section describes a noise tolerant way of deriving the cell structure from a flow field by following the motion of tracker particles in the time-reversed flow field.

\subsection{Data Preparation}

In order to show the supergranulation pattern and its time variation in detail, large amounts of high-resolution photospheric velocity data are required. This may be obtained by tracking the motion of the granules as they are advected by the supergranular flow fields. Long, continuous time series of data are required in order to see the slow evolution of the supergranular flows, and high-cadence, high-resolution images are required to resolve the granulation patterns. As a result the only possible data source at the present time is the high resolution $(0.6$ arcsec/pixel, one minute cadence) continuum data sets from the MDI instrument on SOHO (Scherrer et al., 1995). The images were processed to get the velocity field by 
tracking the barely resolved granulation signal using the Balltrack method (Potts, Barrett, and Diver, 2003).

The data set used throughout this paper is from a 64 hour, nearly continuous run by MDI from 15-18 January 1997. The run consists of high-resolution continuum, magnetogram, and dopplergram images taken with a one minute cadence. We use a $480 \times 1024$ pixel $(4.8 \times 10.2$ arcmin), 33 hour (2001 frames) subset of the continuum data. This set was used as it gives the longest time series of a large area co-rotating with the Sun that is available at the current time. The continuum data were rigidly derotated at a rate of 0.243 pixels/minute and filtered for $p$ modes using a spatial fourier filter, and the velocity then derived using Balltrack. Residual differential rotation was fitted and removed. The velocity samples obtained were binned in time with bin widths of $2-6$ hours, and spatially smoothed by convolving with a $2 \mathrm{D}$ gaussian, with $\sigma$ ranging from $1-13 \mathrm{Mm}$. The effect of the smoothing radius and time binning on the derived cell patterns is discussed in more detail in Sections 3 and 4. Absolute calibration of the data was achieved by adding a range of known, small offset velocities to the raw granulation data during the derotation operation, and measuring the effect of this in the derived velocity. A discussion of this method and the intrinsic noise within the any measurement of the flow field may be found in Potts, Barrett, and Diver (2003).

\subsection{Overview}

The images in Figure 1 show the steps in generating images of the supergranular pattern. Figure 1(a) shows the raw velocity field derived from a Balltrack analysis. Some cell structures, particularly the strong ones, are visible, but the cell boundaries are indistinct. Producing a divergence map (outflow regions in red) of the data as shown in Figure 1(b) helps to clarify the situation somewhat, although the cellular structure is still not clear

Part of the problem is that the data are intrinsically noisy: aside from observational errors, the motion itself has a stochastic element due to the turbulent nature of the small-scale flow. Any local method to find the inflows and outflows that requires taking the spatial derivative of the data is dominated by the small-scale features, at the scale used to smooth the data. This can be overcome by analysing the integral effect of the flow, as is usually done by using "cork" like tracking particles. We use the fact that the flow patterns are asymmetric: mostly point-like sources going to line-like sinks. To exploit this asymmetry we take a regular array of starting points and send tracking particles flowing in the opposite direction to the streamlines. All trackers that end up at the same final area (corresponding to the cell upflow region) must be part of the same convection cell.

\subsection{Method in Detail}

1. Take the initial velocity field, (see Figure $1(\mathrm{a})) \mathbf{v}(x, y)$, reverse the flow direction, and normalise to the maximum speed of the flow field $\mathbf{v}_{\mathbf{n}}=-\mathbf{v} / v_{\max }$. It is important that the mean velocity of the data set (normally dominated by the rotation and differential rotation of the Sun) is very much less than the flows due to the supergranules: we depend on the convergence of the tracers at the upflow regions of the cells, so careful derotation of the dataset is important. It may be useful to subtract the mean velocity, or a fitted differential-rotation 


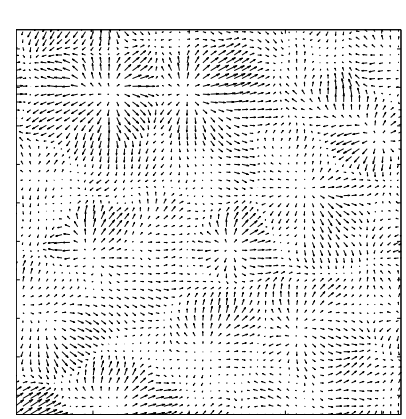

(a) velocity arrows

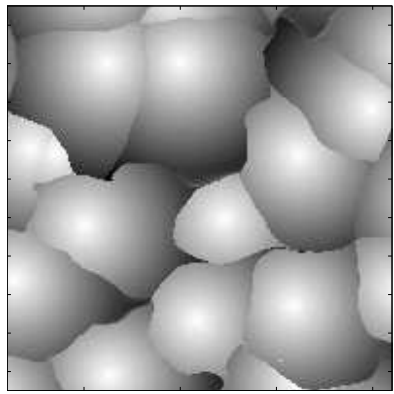

(d) total distance travelled

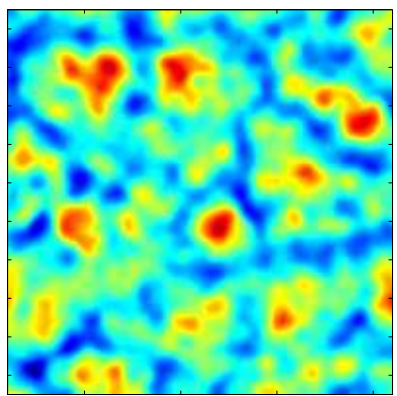

(b) local divergence of flow

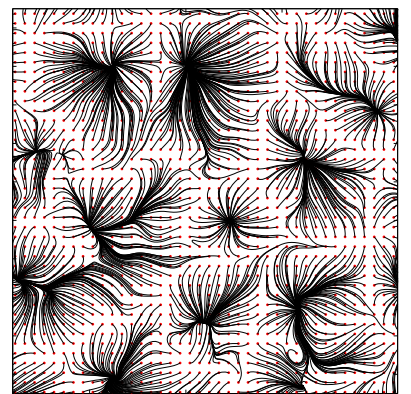

(c) paths of test particles

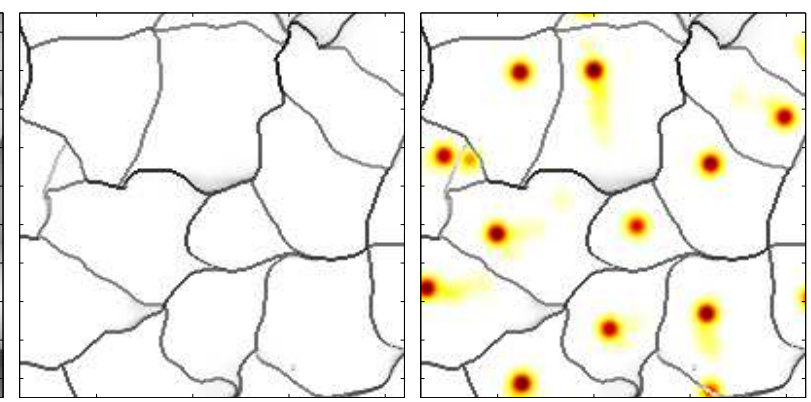

(e) convection cell boundaries

(f) boundaries and upflows

Figure 1. Stages in the identification of the cell boundaries. The data shown are a $2 \times 2$ arcmin $(90 \times 90 \mathrm{Mm})$ region near disk centre under quiet Sun conditions. The velocity field is averaged over three hours, and spatially smoothed by convolution with a $2 \mathrm{D}$ gaussian with radius $\sigma=1.75 \mathrm{Mm}$

correction of the whole flow field from the data at this point to avoid artifacts. See Section 5 for more details.

2. Make a regular array of starting points at whatever final resolution is required for the cell structure. More points will make for a higher resolution, but take longer to calculate

3. Advect the test points with the reversed flow field. The tracks for a low resolution subset of start points are shown in Figure 1c. We use a simple second-order predictor-corrector method for efficiency. Choose a maximum step comparable to the correlation length of the velocity data for maximum efficiency. For maximum clarity enough time steps should be given for a test particle to travel from the edge of a cell to its centre. This process can be made numerically more efficient by the non-linear scaling of the velocity field, so that the particle takes less time to escape from the slow moving edges of the cell. One way to do this is to raise the speed to a power less than unity, while maintaining the direction, for example $\tilde{\mathbf{v}}_{\mathbf{n}}=\hat{\mathbf{v}}_{\mathbf{n}} v_{n}^{0.5}$.

4. Taking the set of the final positions $\left(x_{f}, y_{f}\right)$ of tracer particles mapped onto a grid of their initial positions $\left(x_{i}, y_{i}\right)$, all particles that lie within the same cell will all record the same value of final position; adjacent particles that travel to different cells will record a different final position. Hence such a grid will contain regions in which the values change discontinuously. The gradient of this 
data grid will then reveal the cell boundaries. The quantity $\beta$ expressed below quantifies this process:

$$
\beta=\left[\left(\frac{\mathrm{d} x_{f}}{\mathrm{~d} x_{i}}\right)^{2}+\left(\frac{\mathrm{d} y_{f}}{\mathrm{~d} y_{i}}\right)^{2}\right]^{\frac{1}{2}}
$$

A plot of a suitably normalised value of $\beta$ is shown in Figure 1(e), clearly showing the cell boundaries. It is an exceptionally low noise measurement of the local divergence of the flow.

A property of a cell derived in this manner is that all the tracking particles end up in a similar area, which is the centre of the upflow for the cell. This is shown by the red blobs in Figure 1f. Notice that the distance travelled by the tracking particles is a minimum at these points, as can be seen in Figure 1d. These blobs are a smoothed image of the spatial density of the final positions of the tracking particles, so each one represents a separate upflow region. To find which cell any point on the surface belongs to, simply find out which of these regions the tracking particle ends up nearest to. The area of any cell is proportional to the number of tracking points that travel to this final location. In a movie which can be found in the electronic supplement (Note to editor: link to supergran_colour.avi in the ESM), and also at Potts (2007), each cell, as identified by the upflow regions, has been given a different random colour, and their time evolution can be clearly seen. The change in area of a few selected cells over time is shown in Figure 6, described in more detail in Section 4.3.

\section{Application to Test Data}

In order to test the accuracy of the algorithm, it was run on test data with a known cell structure. The results were compared with the commonly used "watershed basin" algorithm (DeRosa and Toomre, 2004; Hagenaar, Schrijver, and Title, 1997), which does a local minimum search in the divergence field of the flow. The test data were made to have similar properties to observations of photospheric velocity fields, at a resolution equivalent to the of the high resolution output from SOHO/MDI. First, a velocity potential $(\phi)$ was made by producing a Voronoi tessellation of cells from randomly placed generator points, with the value inside the cells a function of the distance to the cell centre. The flow pattern was then obtained by taking the $x$ and $y$ gradient of the potential field, and smoothed by convolving with a gaussian kernel with $\sigma=3$ pixels to represent instrument effects. Noise was then added in variable proportion and the data smoothed again by convolution with a gaussian kernel of variable width.

The response of our supergranulation-finding algorithm is summarised in Figures 2 and 3, along with the performance of the watershed basin algorithm, for comparison.

In Figure 2(a), the performance of the algorithm is tested as the noise:signal ratio is increased. The top row shows the divergence of the velocity field, the centre row shows the cell structure recovered by our velocity-based algorithm, and the bottom row shows the results from the divergence-based algorithm. It is clear that our velocity-based algorithm has very high immunity to noise, in comparison 

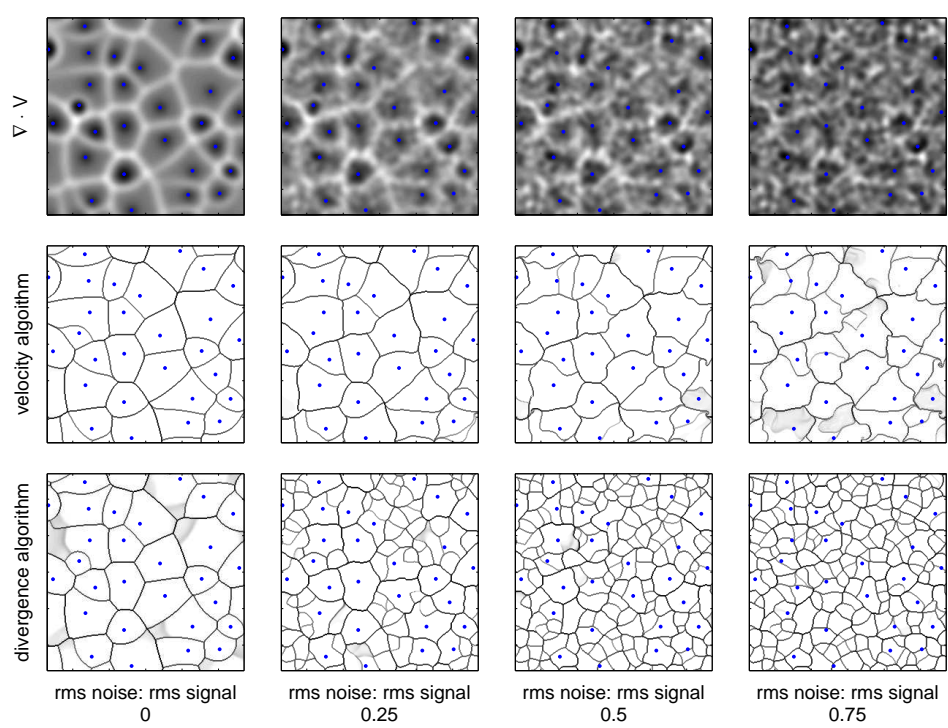

(a) increasing noise at smoothing radius $\sigma=4$ pixels
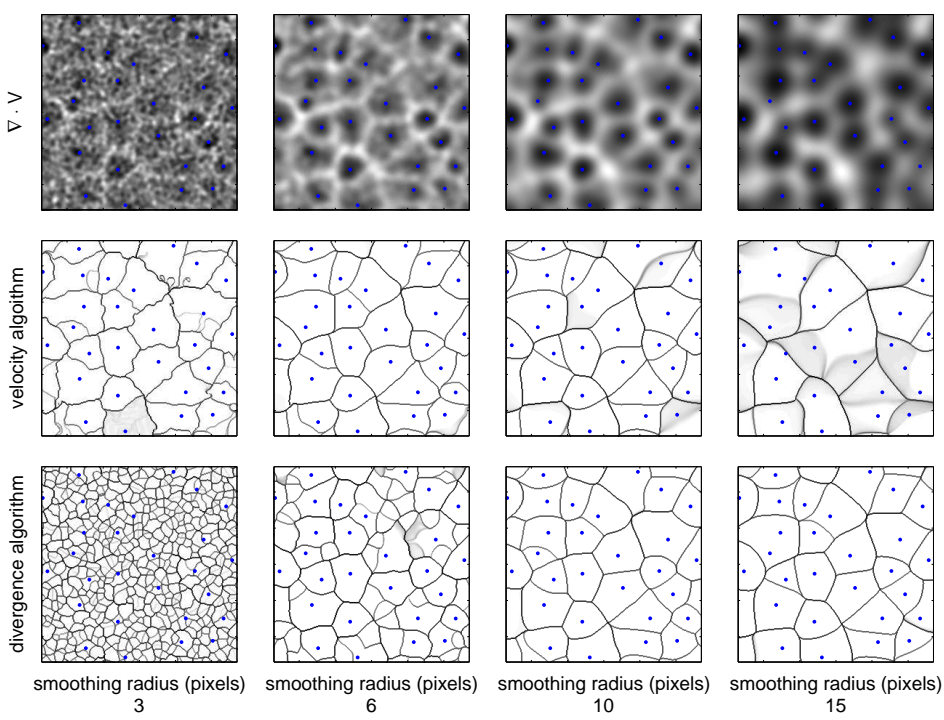

(b) increasing smoothing at rms noise: $r m s$ signal ratio $=0.4$

Figure 2. Test data with increasing noise (top) and increasing smoothing (bottom). In each figure the top row is the divergence field, and the results from our velocity-based segmentation algorithm are shown in the centre row, and the results from the watershed-basin algorithm in the bottom row. The blue dots give the true outflow centres of the cells. Where gray shading occurs on the cell boundary maps it is because the boundary is not clearly defined, usually because the velocity is too small or noisy. 

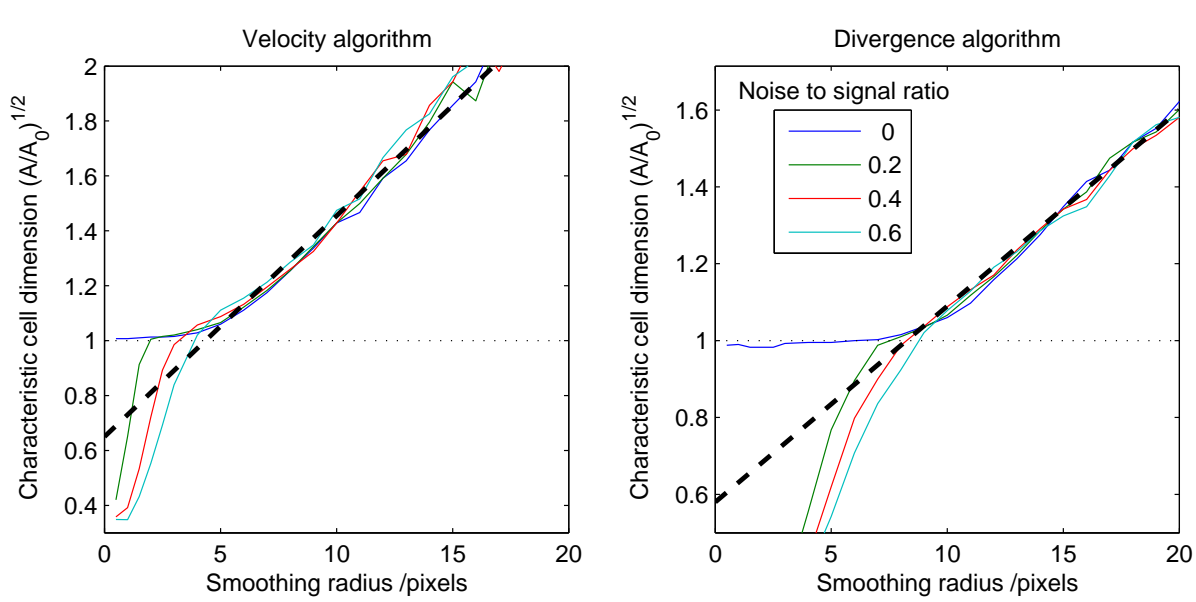

Figure 3. Recovered characteristic cell dimension as a function of smoothing radius for the velocity-based method (left) and the divergence-based method (right), for different noise:signal ratios. The dashed line shows the misleading result of a linear extrapolation from high smoothing radii to zero smoothing

with the divergence-based methods, returning consistent and accurate results, even when the smoothed RMS noise amplitude is similar to that of the data. Note that the left-most plots are the zero noise case, where both algorithms recover the true cell structure of the noise-free test data, and so acts as a reference. In Figure 2(b), the effect of increasing smoothing on noisy data is presented. The test data in this case had fixed amplitude noise, equivalent to a rms noise:signal ratio of 0.4 when smoothed with a radius of four pixels. As the smoothing level is varied, the divergence-based algorithm gives much better results at higher smoothing radii. These effects are compared in more detail in Figure 3, where the effect of derived cell size as a function of smoothing is analysed.

The increased sensitivity to noise of the divergence algorithm can be explained by the effect of differentiating the noise on the signal. If the noise has a flat spatial spectrum, the result of spatial differentiation is to produce a noise signal where the amplitude is proportional to the wavenumber $(k)$, resulting in greatly enhanced short-wavelength noise. The result of this is to introduce many spurious sources and sinks into the divergence field, as can be seen in Figure 2(a). The velocitybased algorithm, in contrast, integrates the flow along streamlines, mitigating the effect of small-scale noise.

The detailed effect of smoothing is shown in Figure 3 for the two methodologies. Both algorithms show an average increase in the returned cell size as the smoothing radius is increased. The true result is a value of unity on the $y$-axis. Our velocitybased algorithm fares much better at low smoothing radii, but becomes more than $20 \%$ inaccurate as the smoothing radius exceeds seven pixels. The divergence algorithm, conversely, has a complementary performance, showing poor accuracy up to a smoothing radius of seven pixels, and above 15 pixels. Both algorithms show a linear trend in derived cell size as a function of smoothing.

All real data have an element of smoothing from a variety of unavoidable sources: for example, instrumental effects, seeing, and noise-reduction algorithms. To mitigate these effects in the data reduction, one approach that was first used by 


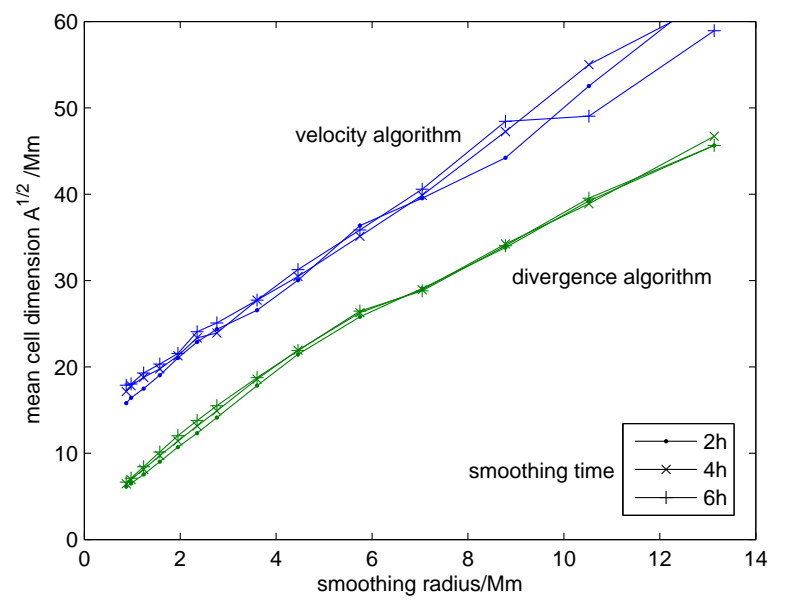

Figure 4. The effect on cell size of changing smoothing radius and time applied to high resolution velocity fields derived from MDI continuum data

Hagenaar, Schrijver, and Title (1997), and was also used by DeRosa and Toomre (2004) is to smooth the data at different smoothing scales, and then extrapolate back to infer the true result that corresponds to the zero-smoothing case. This assumes that the effect of smoothing is linear in the returned cell size. Since we have test data, we can assess the efficacy of this technique. The results of this operation are shown by the thick dashed lines in Figure 3. It is clear from this that the trend of recovered feature size being proportional to smoothing radius is only linear at large smoothing radii, where coincidentally the watershed-basin algorithm works well. However, using this linear regime on this test data to extrapolate to the zero-smoothing radius case significantly underestimates the true, underlying structure size for the test data, mainly because the linear behaviour is not valid at small smoothing radius. This result leads us to conclude that mean cell diameters obtained by extrapolation in this way are not necessarily secure.

\section{Application to Real Data}

Here we show how this noise-tolerant and accurate supergranular cell finder can be applied to real solar data, and exploited to reveal additional properties of the photospheric flow field. The data set used here is the same as that in Section 2.1.

\subsection{Cell Sizes}

In order to measure the mean cell size in the real data, the same procedure was followed as described for the test data in Section 3, where the data were smoothed over a range of radii. The results are shown in Figure 4, where the derived mean cell dimension is shown as a function of smoothing radius, for the two algorithms. There is a clear difference between these results and those of the test data: there is no sharp fall-off at small smoothing radii. This reflects the fact that there is no definite 

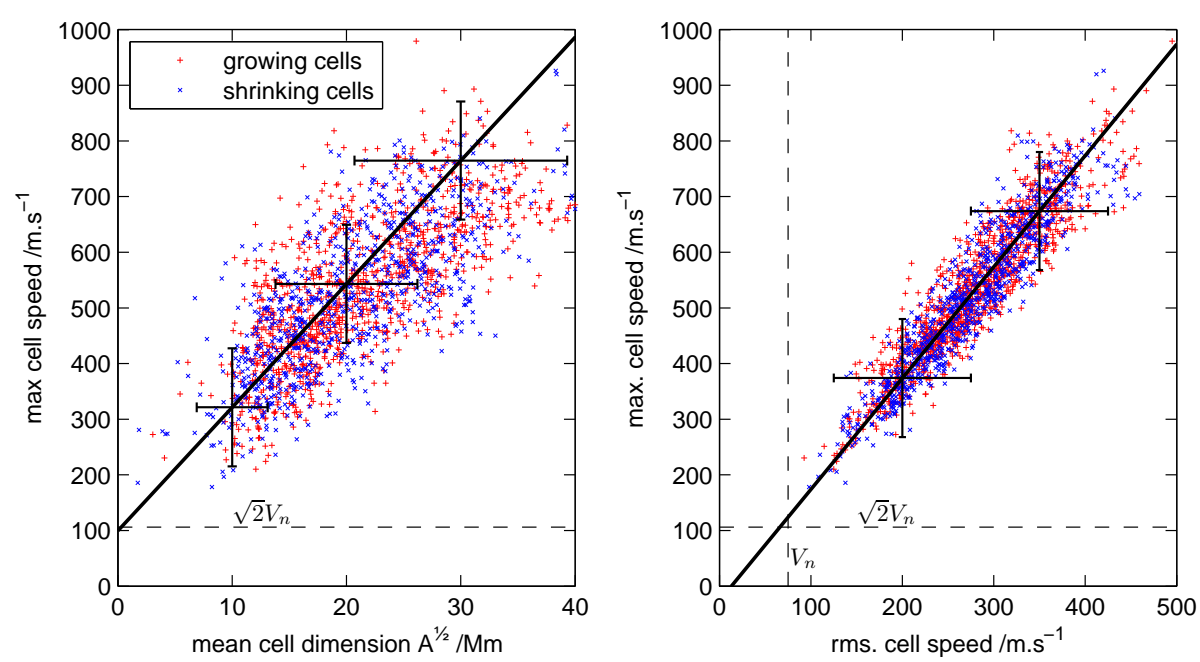

Figure 5. Relation between the size of supergranular cells and their velocities for approx. 2000 cells. The left plot shows the peak speed in each cell versus the cell dimension, with a linear relation fitted, taking into account the indicated velocity and dimension errors. The right hand plot shows the relation between the cell rms velocity and the peak velocity, again with a linear fit

minimum scale for the features in the real data in comparison to the test data. The next clear effect is that there is almost no significant variation in the results at different smoothing timescales, showing that the supergranulation timescale must be significantly larger than our smoothing times, as observed by other authors (Del Moro et al., 2004; DeRosa and Toomre, 2004; Hagenaar, Schrijver, and Title, 1997).

For the divergence results, at smoothing radii less than $6 \mathrm{Mm}$, the characteristic cell size is nearly proportional to the smoothing radius. This is the result that would be obtained from smoothing random noise that had no true cell structure, showing that the algorithm is dominated by noise in this region. Thereafter the results show a similar linear form to that observed by DeRosa and Toomre (2004) at these smoothing radii, and when extrapolated back to zero smoothing yields cell dimension of around $8 \mathrm{Mm}$, smaller than that from previous analyses of lower resolution data.

The velocity algorithm yields larger cell dimensions for a given smoothing radius, and remains linear over the whole smoothing range. Unlike the divergence results, the velocity method shows evidence of real structure at low smoothing radii (right down to an unprecedented spatial resolution of $0.9 \mathrm{Mm}$ with only two hours of temporal smoothing). The short extrapolation to zero-smoothing gives a cell dimension of approximately $15 \mathrm{Mm}$, in broad agreement with aforementioned previous studies in the literature. As the data become progressively noisier at smaller smoothing radii, this result gives a lower limit for the characteristic cell dimension, since noise can only decrease the observed dimension. 


\subsection{Cell Internal Speeds}

With an easy way to delimit the parts of the photosphere that belong to a particular cell, it is simple to derive other useful data. One such quantity is the speed profile within supergranular cells. The data that was used in this section is the 33-hour set described in Section 2.1, smoothed with $\sigma=1.75 \mathrm{Mm}$ and three hour time binning. This smoothing was chosen to be small enough such that the cells recovered were much larger than the smoothing radius, ensuring that the smoothing did not significantly affect the shape or internal velocity profile of the cells.

Following the analysis of Meunier et al. (2007) and Krishan et al. (2002), we looked for a power-law relation between the cell size and internal speeds. For this we selected cells that could be tracked with consistent areas for at least three hours (see Section 4.3), to be certain that the cells were well defined. This left us with approximately 2000 cells, with approximately $55 \%$ growing and $45 \%$ shrinking.

When looking for a relation such as this, the effect of the errors in the measured quantities must be taken into account. We performed the analysis on both the rms speed within the cells (errors add in quadrature to the true data) and the peak speed (errors add linearly to increase the peak). To estimate the errors in the speed data we use the results of the analysis in Potts, Barrett, and Diver (2003), which examines the velocity of the individual granules that are just resolved in MDI continuum images, and looks at the statistical effect of smoothing that data. From this we get a minimum rms noise of $75 \mathrm{~m} \mathrm{~s}^{-1}$ for the smoothing parameters used here. In reality the noise may be greater than this as this method assumes perfect tracking of every granule. The "noise" in the characteristic cell size (the square root of the area) is related to the variation in the shapes of the cells. The cells as observed range from roughly circular (well defined radius) to the more commonly observed cusped shapes with aspect ratios ranging from square to around 2:1 (see Figures 1 and 6 for examples). The centre-edge distances in a 2:1 rectangle vary from 0.6 to 1.4 times that of the radius of a circle with the same area; this variation was used as the maximum noise. The power law was then fitted using an iterative scheme, taking into account the point by point errors. To estimate the accuracy of the fits each was performed repeatedly on a random selection of $50 \%$ of the data.

Our results, taking into account these errors, give a considerably stronger sizespeed relation than that observed in the literature. For a rms speed error of $75 \mathrm{~m} \mathrm{~s}^{-1}$, combined with a size error of $0.3 \sqrt{A}$ we get a power law with index 0.72 for the rms velocity and 1.06 for the maximum velocity. If we choose larger errors of $100 \mathrm{~m} \mathrm{~s}^{-1}$ for the speed and $0.4 \sqrt{A}$ for the radius, we get indices of 0.96 for the rms. speed and 1.24 for the maximum speed. The error on the individual power law fits was \pm 0.025 . There was no significant difference in the results between the growing and shrinking cell populations.

Note that these values are both close to, and centred around, an index of unity, suggesting that there may be a simple linear relation between cell size and internal speeds. We explore this in Figure 5, which shows the maximum speed within a cell as a function of the cell dimension, and the relation between the maximum speed and the rms. speed within the cell. The error bars shown are representative for those used for all the data points. We chose an rms noise of $75 \mathrm{~m} \mathrm{~s}^{-1}$ and a cell size error of $0.3 \sqrt{A}$ as described above. The lines are two-parameter, iterative straight-line fits, taking into account the point-by-point errors. In the left hand 
plot, if the cell velocity were proportional to the cell size, it would be expected that the $y$-intercept of the fit would be equal to the peak value of the noise, roughly $\sqrt{2}$ times larger than the rms noise, indicated by the horizontal dashed line in both plots. The line is a very close fit to this, with the distribution of points in line with the chosen errors indicating that our choice of noise amplitude is reasonable. In the right-hand plot, the relation between the rms speed and the maximum speed within the cells is shown, fitted in the same manner. Notice that the error bars in this case appear too large; this is due to the fact that the maximum and rms speed errors are correlated. Again, a linear fit seems reasonable.

This result substantially disagrees with the findings of Krishan et al. (2002) which found a weak power law with index 0.34, and interpreted this in terms of the solar convective motions obeying the Kolmogorov hypothesis for a turbulent medium. The main reason for this is probably due to bias in their subjective selection method where a small number of unusually large cells were picked by eye. Our work should be free of this as all consistent cells within the analysis region were chosen, resulting in a much larger sample free of selection effects.

Our results are much closer to those of (Meunier et al., 2007), where a index of 0.66 was found. This is very near to our lower limit of $0.72 \pm 0.025$, although sill considerably less than our average value of unity. The probable reason for this is the effect of the error analysis, which significantly steepens the power-law fit. Another factor is that the cells found in this study could all be much larger than the smoothing used, due to the noise tolerance of the cell-finding algortihm, avoiding any systematic errors due to smoothing artifacts.

The conclusion from this is that is that a simple linear relationship between cell size and internal speed matches the data within the limitations of the measurement errors. There is also no difference between the behavior of growing or shrinking cells, also there are approximately equal numbers of both. This linear behavior suggests that cells are self-similar, with the same internal velocity distribution, independent of size. The implications of this for the energetics of the convectional process need to be addressed.

\subsection{Tracking Supergranular Cells over Time}

Identifying a cell at a particular time is interesting, but even a random velocity field, appropriately smoothed, would show some cell-like structures. Confidence that the cells obtained are real physical phenomena is gained by showing the evolution of the supergranular pattern over consecutive times using time-separated data sets. If the data can be processed this way, the results allow the growth and decay of the patterns to be monitored, as is used in the previous section.

There are many problems with tracking supergranular cells over time, the most significant of which is that the cells are continuously splitting and merging. We have developed an algorithm that tracks the upflow points of the cells though time (see Figure 1(f)). This allows us to track the boundary of individual cells over long time periods (limited only by the available data), and stores all of the branching and merging events from the entire data set. All other data about the cells are also stored, such as the cell area, velocities, centroid, and upflow centre.

The evolution of some representative cells found by the algorithm over a 30 hour period are shown in Figure 6. Cell A is an unusual cell that grows rapidly over a 15hour period from the intersection of several cell boundaries. It is worth noting that 
A
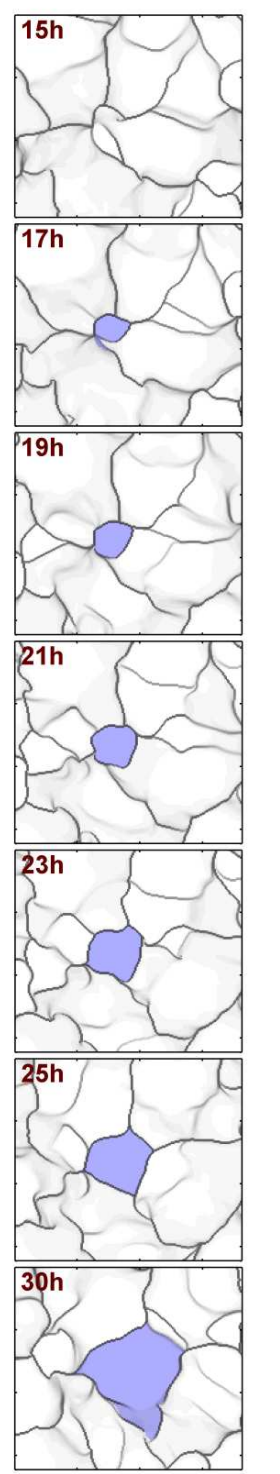

B
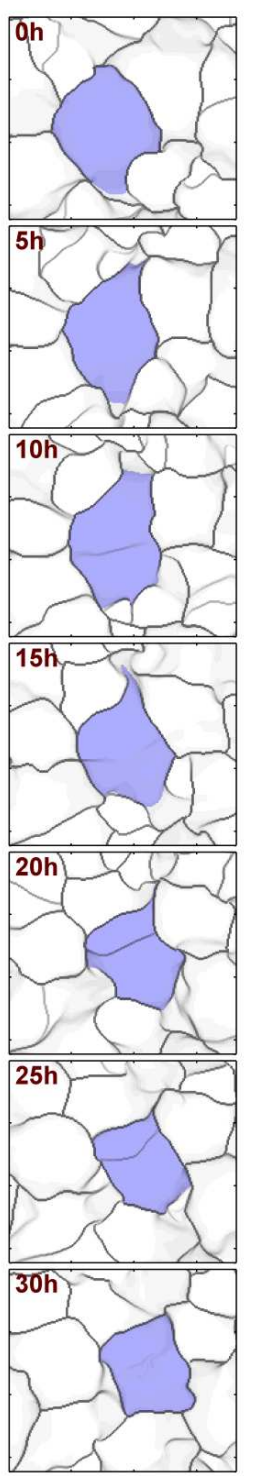

C
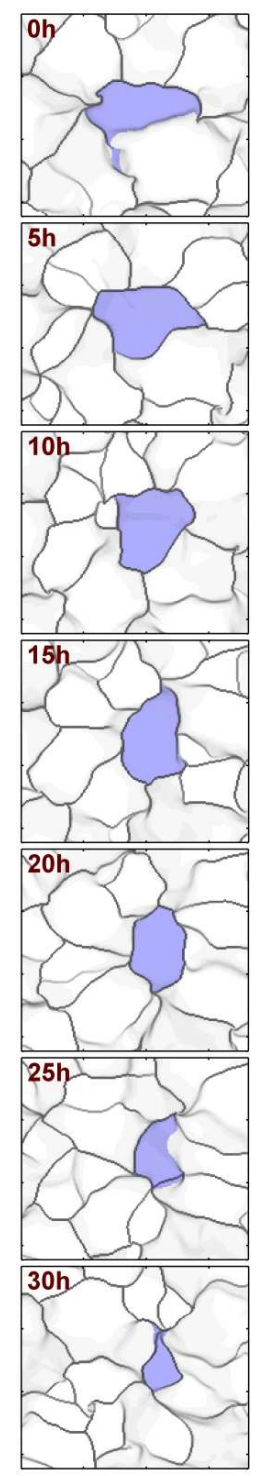

D
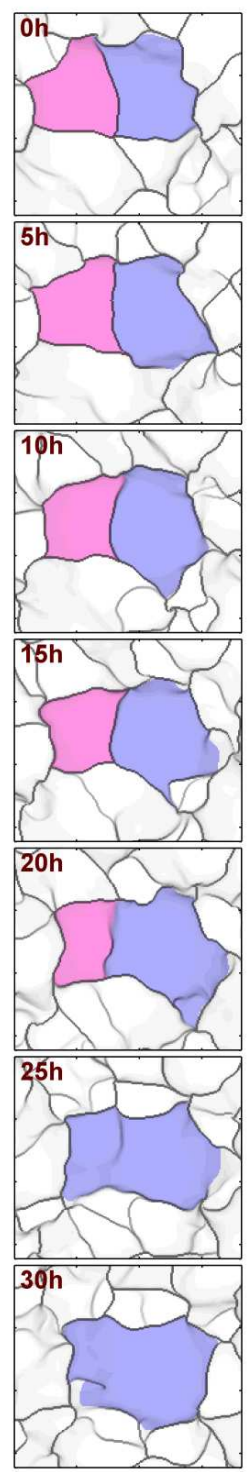

Figure 6. Evolution of a selection of supergranular cells over a 31-hour period. Each of the images is $110 \times 100$ arcsec. Each column shows a cell at different intervals after it was first observed, shown in hours at the top-left of each frame. This data set has been corotated with the Sun to set the mean plasma (granule) velocity to be zero, yet note the significant westwards motion of the supergranules, showing that they rotate faster than the fluid that forms them. A movie showing the time evolution of the entire data set over a 36 hour period can be found in the electronic supplement (Note to editor: link to supergran_colour.avi). (Potts, 2007). 
this cell is unusually free of magnetic field as it grows. Cell B stays very stable over the 30-hour period. Cell C slowly breaks up and shrinks over the 30 hour period. Cells D are a pair of fairly large cells that merge to produce a single cell.

Note that although this data set was derotated so that the mean equatorial velocity was zero, there is a clear solar westward motion of all of the cells, corresponding to a rotation rate which is around $30 \mathrm{nHz}$ faster than the rotation of the granules themselves. This is a well known phenomenon, (Thompson et al. (2003), Beck (2000)), but as yet there is no clear consensus in the literature as to the underlying physics.

\section{Cautionary Notes}

Whenever a continuous process is studied by looking at discrete time steps, the frequency of observation and the amount of smoothing in time can greatly influence the results. Such influences can be highly significant in the sort of data processing addressed in this article: the flow field itself is obtained by spatially smoothing the small-scale granular motions. This means that any derived supergranulation flow field obtained has been implicitly convolved with whatever temporal and spatial smoothing was used in the observation. This problem is ubiquitous in any measurement of supergranulation, whether from dopplergrams, or even from the measurements of the chromospheric network, where the lifetime of small magnetic elements imposes a natural timescale on the data.

It is also important to be very careful about how the velocity data used to derive the cells is derotated, due to the small values of the velocity field $\left(\approx 300 \mathrm{~m} \mathrm{~s}^{-1}\right)$ in comparison to the rotation rate of the Sun (equator speed $\approx 2 \mathrm{~km} \mathrm{~s}^{-1}$ ). For a weak supergranule, the outflow speed near the edges is very small, so the apparent position of the lanes can change considerably due to a small derotation error. Small cells can completely disappear if the rotation offset is larger than their peak velocity. One way to help prevent this is to subtract the mean velocity with differential rotation corrections from the velocity field as we have done here.

\section{Conclusions}

We have developed a method for automatically identifying supergranulation cells, including an accurate measure of the position of lanes between cells and the upflow centre of the cells, without resorting to numerical differentiation of the data.

Since our method can work at exceptionally small smoothing radii, extrapolation to the zero-smoothing radius case is more secure than for conventional algorithms, which tend to need high smoothing to avoid domination by noise. This makes out method particularly well suited to the new generation of high resolution solar data.

Our key physical result from this paper is that there appears to be a simple linear relation between cell size and cell internal velocity, rather than the power law usually suggested. In addition we can track the cells over considerable times, limited only by the source data, and from this observe in detail their evolution. Due to the noise tolerance of the algorithm, the smoothing radius required is only one 
to two Mm, much smaller than the size of supergranular cells, allowing structure identification in unprecedented detail.

There are many other applications for which the process outlined in this paper will be useful. For example the the problem of measuring any temperature differential across the cells will be greatly reduced by having accurate positions for the cell outflow and inter-cell lanes (e.g. Meunier, Tkaczuk, and Roudier (2007)). Another example is the study of the small-scale energetics of magnetic elements, (Potts and Diver, 2007) whose motion is dominated by the supergranular flows. Accurate measurements of the statistical properties of supergranulation, both over the supergranular evolution timescale and over the solar cycle will help expand our knowledge of this poorly understood process.

The study of small scale energetics will also benefit greatly from this method, particularly small scale magnetic interactions which are dominated by the solar surface flows. As high resolution Hinode data becomes available this will become a very interesting area to study.

\section{Acknowledgements}

We would like to thank Lyndsay Fletcher and Hugh Hudson for helpful discussions that have considerably improved this paper.

This work was funded in the UK at Glasgow University by PPARC rolling grant number PP/C000234/1.

\section{References}

Beck, J.: 2000 Solar Phys. 191, 47-70.

Del Moro, D., Berrilli, F., Duvall, T.L., Kosovichev, A.G.: 2004, Solar Phys. 221, 23-32.

DeRosa, M.L., Toomre, J.: 2004, Astrophys. J. 616, $1242-1260$.

Hagenaar, H.J., Schrijver, C.J., Title, A.M.: 1997, Astrophys. J. 481, 988-995.

Hart, A.B. Mon. Not. Roy. Astro. Soc. $11417-38$

Krishan, V., Paniveni, U., Singh, J., Srikanth, R: 2002, Mon. Not. Roy. Astro. Soc. 334, $230-232$

Leighton, R.B., Noyes, R.W., Simon, G.: 1961, Astrophys. J. 135 474-499.

Lin, H., Kuhn, J.R.: 1992, Solar Phys. 141, 1-26.

Meunier, N., Tkaczuk, R., Roudier, T., Rieutard, M: 2007, Astron. Astrophys 461, 1141-1147.

Meunier, N., Tkaczuk, R., Roudier, T.: 2007, Astron. Astrophys 463, 745-753.

Potts, H.E., Barrett, R.K., Diver, D.A.: 2003, Solar Phys. 197, 69-78.

Potts, H.E.: 2007 http://www.astro.gla.ac.uk/users/hugh/supergranulation

Potts, H.E., Diver, D.A.: 2007, Solar Phys. (in press) (DOI: 10.1007/s11207-007-9021-7)

Scherrer, P.H., Bogart, R.S., Bush, R.I., Hoeksema, J.T., Kosovichev, A.G., Schou, J., Rosen-

berg, W., Springer, L., Tarbell, T.D., Title, A., Wolfson, C.J., Zayer, I., MDI Engineering Team: 1995, Solar Phys. 162, 129-188

Thompson, M.J., Christensen-Dalsgaard, J., Miesch, M.S., Toomre, J.: Ann. Rev., 41, 599-643 